Check for updates

Cite this: RSC Adv., 2018, 8, 18118

\title{
Nano-hydroxyapatite polymeric hydrogels for dye removal†
}

\author{
Kokkarachedu Varaprasad, (D) *a Dariela Nunez, ${ }^{* a}$ Murali Mohan Yallapu, ${ }^{\text {b }}$ \\ Tippabattini Jayaramudu, (D) ${ }^{c}$ Elizabeth Elgueta ${ }^{a}$ and Patricio Oyarzun ${ }^{d}$
}

\begin{abstract}
Herein, two kinds of nano-hydroxyapatite were synthesized from Clam and Magellan shell by wet chemical precipitation method. Mainly, carboxymethyl cellulose/acrylamide/nano-hydroxyapatite composite hydrogels were developed via a free-radical polymerization process and investigated as a sorbent for Acid Blue 113 (AB) from aqueous $A B$ solution. The swelled and kinetic behaviours of hydrogels were investigated using a gravimetric method. The swelling properties of the CMC-AM-hydrogels were influenced by the calcium electrolytes $\left(\mathrm{Ca}^{2+}\right)$ content in nano-hydroxyapatites. The diffusion coefficient value increased with the increase of nano-hydroxyapatite content in the CMC-AM/nHA-CS $(0.22353-$ $\left.0.27681 \mathrm{~cm}^{2} \mathrm{~s}^{-1}\right)$ and CMC-AM/nHA-MS $\left(0.22377-0.29737 \mathrm{~cm}^{2} \mathrm{~s}^{-1}\right)$ hydrogels. The mechanism of water diffusion was found to be anomalous transport. The CMC-AM/nHA-MS hydrogels showed high $A B$ absorption efficiency and adsorption capacities. These results explained that the nano-hydroxyapatites of Magellan shells based hydrogels are attractive nanocomposite hydrogels for the adsorption of dye in the water purification applications.
\end{abstract}

Received 4th March 2018

Accepted 8th May 2018

DOI: 10.1039/c8ra01887a

rsc.li/rsc-advances

\section{Introduction}

The development of nano-hydroxyapatite from mollusk shells is attractive in several fields, such as the removal of pollutants from wastewater. In addition, the use of nano-hydroxyapatite in combination with polysaccharide based biomaterials is a promising alternative in environmental technology applications. Effluents from many industries contain various kinds of pollutants, particularly dyes which can create water pollution damaging the aesthetic nature of the environment. ${ }^{1}$ Removal of hazardous stains from contaminated water is environmentally challenging because these compounds may produce health problems for living systems due to their toxicity, low degradability (due to complicated structures) and their persistent bioaccumulation. ${ }^{2,3}$ Techniques such as adsorption, photocatalysis, electrochemical and other methods have been used for removal of a variety of dyes from polluted water. ${ }^{3}$ Among these techniques, an adsorption method is simple, cost-

${ }^{a}$ Centro de Investigación de Polimeros Avanzados, CIPA, Avenida Collao 1202, Edificio de Laboratorios, Concepción,Chile.E-mail: prasad@cipachile.cl; d.nunez@cipachile. $c l$

${ }^{b}$ Department of Pharmaceutical Sciences, Center for Cancer Research, University of Tennessee Health Science Center, Memphis, TN 38105, USA

'Laboratory of Material Sciences, Instituto de Quimica de Recursos Naturales, Universidad de Talca, Talca, Chile

${ }^{d}$ Facultad de Ingeniería y Tecnología, Universidad San Sebastián, Lientur 1457, Concepción 4080871, Chile

$\dagger$ Electronic supplementary information (ESI) available. See DOI: $10.1039 / \mathrm{c} 8 \mathrm{ra} 01887 \mathrm{a}$ effective, and a direct analytical process. In this method, several hydrophilic polymeric hydrogels were used for purification of wastewater due to its three-dimensional structures, large specific surface area and their swelling and de-swelling properties. ${ }^{4,5}$ However, these characteristics are dependent on the composition of hydrogels including removal of dyes from polluted water. Lately, significant attention has been paid to polysaccharide hydrogels for the discharge of pollutants from the aqueous solution. ${ }^{4}$ Sodium carboxymethyl cellulose is one of the linear anionic (polyelectrolyte) polysaccharide. It is an inexpensive, biodegradable/biocompatible natural polymer which can enhance the swelling and absorbent characteristics of hydrogels, due to its strong hydrogen bonding and multiple carboxyl groups..$^{6-8}$ Further, it has been employed in the preparation of hydrogels for a variety of applications, including drug delivery, tissue engineering, antibacterial, dyes and ionic adsorption. ${ }^{9}$

To further increase the adsorption capacity of hydrogels, nanoparticles have been encapsulated into the hydrogels. Among many nanoparticles, hydroxyapatite (HA) or nanohydroxyapatite (nHA) is considered one of the inorganic bioceramic. It is recognized as a potential candidate for several applications in biomedical engineering, environmental technology, nanotechnology, chemical engineering, and materials science. $^{10,11}$ Due to its biocompatibility, osteoconductivity, biodegradability and synthetic nanostructures (calcium and phosphorus combined hydroxide) as well as an affinity for polymers. ${ }^{12-14}$ Therefore, hydroxyapatite has been used in the preparation of polymeric composite materials with various 
polymers, such as chitosan, alginate, cellulose, collagen, poly(lactic acid), poly( $\varepsilon$-caprolactone) for engineering applications. ${ }^{\mathbf{1 5}}$ Hydroxyapatite can be synthesized from the waste of fish bones, egg shells, oyster shells, coral, algae and other biogenic sources for respective applications. ${ }^{16}$ The fish industry discards tons of mollusk shells which causes environmental problems in many countries including Chile. The waste materials have been used for landfills. ${ }^{13,17}$ Researchers have been using mussel shell products for paper, rubber, paints and pharmaceutical industrial applications because they have 95-99\% of biogenic aragonite in their mass. ${ }^{18}$ Papadimitriou's group ${ }^{13}$ studied the dye and large adsorption properties of waste mussel shells. Recently, nano-hydroxyapatites were produced from mussel shells to enhance their characteristics for advanced applications. ${ }^{15}$ According to literature, calcium electrolytes $\left(\mathrm{Ca}^{2+}\right)$ ion exchange properties make them accessible in biomedical applications. ${ }^{19}$ Also, HA has demonstrated high efficiency to remove pollutants from the environment. ${ }^{19}$ Khal et al., ${ }^{20}$ has reported hydroxyapatite adsorptive properties. Polyacrylamide with hydroxyapatite composites was also prepared for adsorption of metal ions and proteins. ${ }^{21,22}$ Hou et al. ${ }^{23}$ focused on the absorption properties of the carboxymethyl cellulose (CMC)/ chitosan/HA composite for removing dye from aqueous solutions but not any of the dynamic aspects. Therefore, the objective of this study is to prepare polysaccharide nanocomposite hydrogels using CMC, nano-hydroxyapatite and acrylamide hydrogels via a free-radical polymerization process to remove $\mathrm{AB}$ (a commonly used dye in the textile industry). The nano-hydroxyapatites of Magellan shells (nHA-MS) and nanohydroxyapatites of Clam shells (nHA-CS) were developed by the wet chemical precipitation method. Attenuated total reflectance-Fourier transform infrared (ATR-FTIR) spectroscopy, X-ray diffraction (XRD), scanning electron microscope (SEM) and transmission electron microscope (TEM) techniques were used to identify the chemical structure and morphology of the nHA. The effects of nHA-MS/nHA-CS content in CMC/AM/ nHA on the swelling behaviour and dye adsorption of Acid Blue 113, were investigated.

\section{Experimental section}

\section{Materials and methods}

Sodium carboxymethylcellulose (CMC), acrylamide (AM), N, $N^{\prime}$ methylene-bis-acrylamide (MBA), ammonium persulphate (APS), Acid blue 113 (AB), phosphoric acid were purchased from Sigma-Aldrich, Chile. Clam and Mussel shells were collected from local markets in the province of Concepcion, Bio-Bio, Chile.

\section{Synthesis of nano-hydroxyapatite (nHA)}

For the preparation of nano-hydroxyapatite, two species of discarded mussel shell were used: Clam shell (CS) and Magellan shell (MS) were employed. Both bivalves are native species from Conception in Bio-Bio region, Chile. The discarded shells were ground with a laboratory mill and sieved via a 100 Mesh stainless steel screen to achieve fine shell powder. Furthermore, it was calcined in a furnace at $900{ }^{\circ} \mathrm{C}$ for 3 hours to eliminate the unwanted organic materials present in the shells and obtain $\mathrm{CaO}$ from the $\mathrm{CaCO}_{3}$. The subsequent $\mathrm{CaO}(5.61 \mathrm{~g})$ was hydrated with $100 \mathrm{~mL}$ of distilled water to produce an aqueous solution of $\mathrm{Ca}(\mathrm{OH})_{2}$ and $\mathrm{H}_{3} \mathrm{PO}_{4}(4.5 \mathrm{~mL} / 100 \mathrm{~mL})$ was added drop-by-drop at a flow rate of $2.5 \mathrm{~mL} \min ^{-1}$ using a syringe pump. The reaction vessel was stirred at $1500 \mathrm{rpm}$ at $40{ }^{\circ} \mathrm{C}$ for 40 minutes. Finally, the reaction solution $\mathrm{pH}$ was adjusted to 10 by using $\mathrm{NaOH}$ and stirred overnight at $20 \pm 2{ }^{\circ} \mathrm{C}$. The obtained precipitate was washed three times with distilled water by centrifugation, resuspended and finally dried in an oven at $40{ }^{\circ} \mathrm{C}$ for three days and powdered in a mortar.

\section{Preparation of hydrogels}

The nHA-MS and nHA-CS based composite hydrogels were prepared through free-radical polymerization process of CMC and AM, as shown in Scheme 1.

A typical procedure described as follows. CMC solution was prepared by dissolving $0.10 \mathrm{~g}$ in $5 \mathrm{~mL}$ under stirring condition. $5 \mathrm{mg}$ of nHA-MS in $1 \mathrm{~mL}$ distilled water was added to the

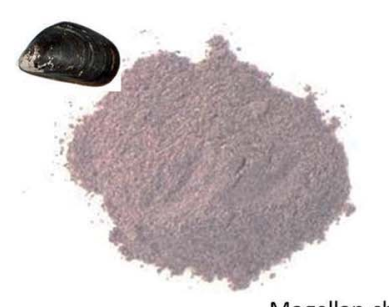

Magellan shel

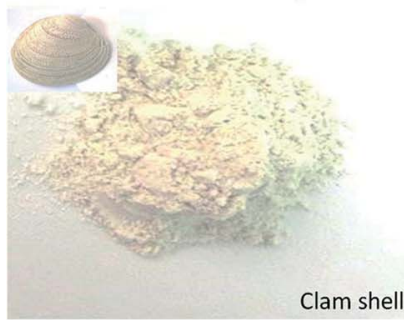

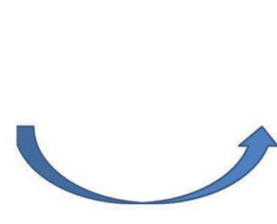

Wet chemical precipitation

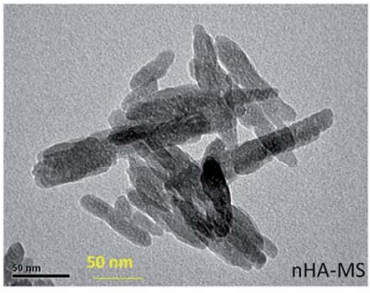

Nano-hydroxyapatite

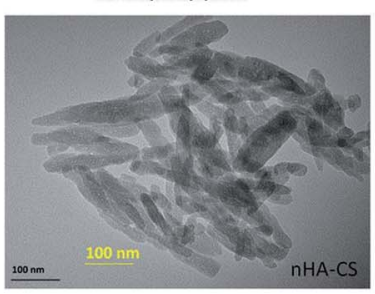

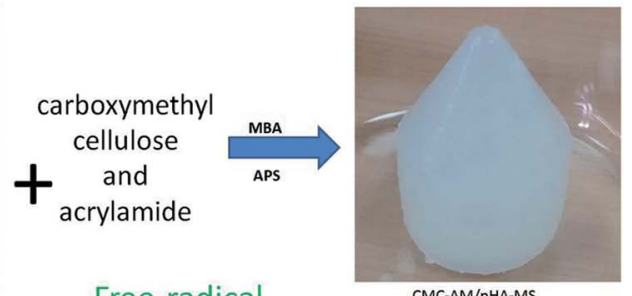

Free-radical polymerization

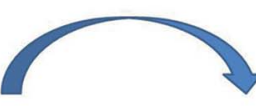

$\underline{100}$

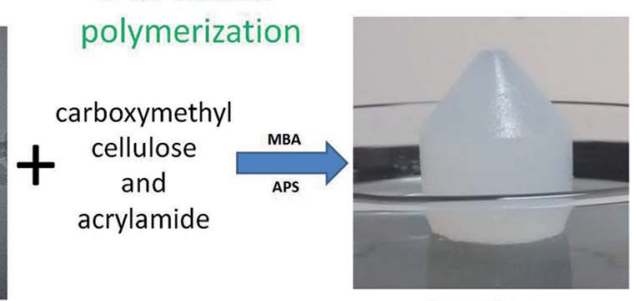

CMC-AM/nHA-CS

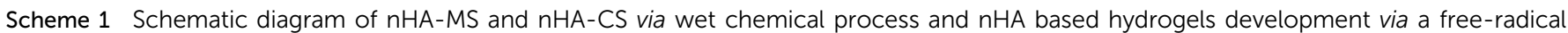
polymerization process. 


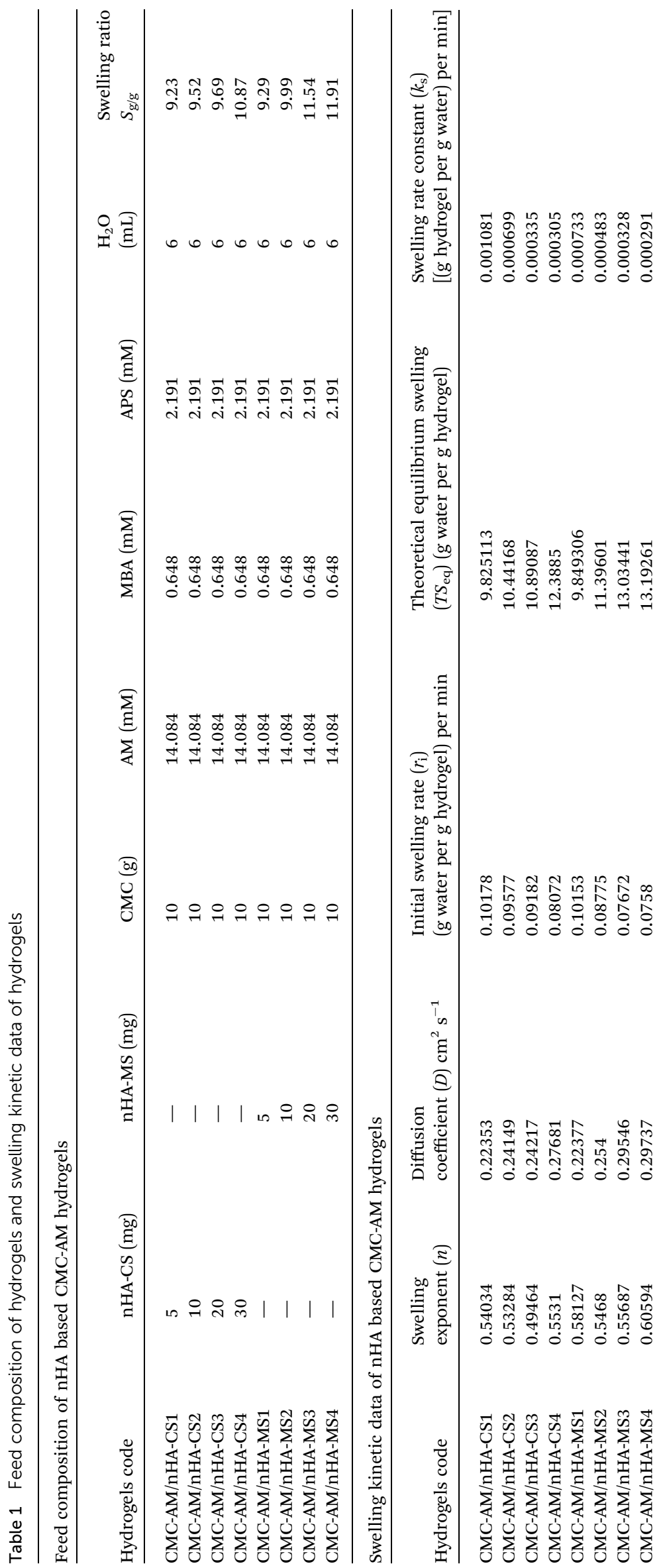


solution above and sonicated for 15 minutes to make a solution with a homogeneous distribution of nHA. Then, $14.06 \mathrm{mM}$ of AM, $0.648 \mathrm{mM}$ of MBA, and $2.191 \mathrm{mM}$ of APS were sequentially introduced into the solution. The reaction solution was stirred for 2 hours to form a hydrogel. The resulting hydrogel was washed with distilled water and immersed in distilled water to remove unreacted components for 24 hours. The entire hydrogel preparation was conducted at $21 \pm 2{ }^{\circ} \mathrm{C}$. Similarly, other hydrogels were prepared following the same procedure. The feed compositions of the composite hydrogels are presented in Table 1.

\section{Physico-chemical characterization}

FTIR spectra of the nano-hydroxyapatites and composite hydrogels were obtained from a Perkin Elmer, UATR two, ATRFTIR spectrometer (Beaconsfield, Bucks, UK) in the wavelength range of between $4000-400 \mathrm{~cm}^{-1}$. Powder X-ray diffraction profile of hydroxyapatites and composite hydrogels was studied with Rigaku diffractometer, with $\mathrm{Cu}-\mathrm{K}_{\alpha}$ radiation at a voltage of $40 \mathrm{kV}$, current of $40 \mathrm{~mA}$, and a scan rate of $0.02^{\circ} \mathrm{s}^{-1}$. The SEM/ EDS (energy dispersive spectroscopy) analysis for nHA was carried out using the JEOL 6460LV scanning electron microscope at a voltage of $10 \mathrm{kV}$. The micro-morphology of composites were observed at a voltage $5 \mathrm{kV}$ with a JEOL JSM-6610LV SEM. The samples were coated with gold by a sputter coater for excellent conductivity. Further gold coated hydrogel samples were air dried. The size and shape of the nHA-CS and nHA-MS nanoparticles were calculated by using the FEI Technai G2 20STWIN, USA transmission electron microscopy (TEM).

\section{Calcium determination in nHAs}

nHA-MS and nHA-CS samples were dried at $105{ }^{\circ} \mathrm{C}$ in the oven for $24 \mathrm{~h}$, then $0.1 \mathrm{~g}$ of the dried sample was diluted in $50 \mathrm{~mL}$ distilled water with $2 \%$ of $\mathrm{HNO}_{3}$. The calcium calibration curve was prepared using $\mathrm{Ca}\left(\mathrm{NO}_{3}\right)_{2}$ standard solution (Merck Co., Germany) at 1, 2, 3, 4 and $5 \mathrm{mg} \mathrm{L}^{-1}$. Atomic absorption spectroscopy (AAS) (PinAAcle 900F, Perkin Elmer) was used for calcium concentration analysis. The flame atomizer was supplied with acetylene and nitrous oxide, and a cathodic lamp was used as the radiation source for calcium measurement. The detection wavelengths were set at $422.67 \mathrm{~nm}$.

\section{Swelling behaviour}

The swelling ratio $\left(S_{\mathrm{g} / \mathrm{g}}\right)$ of composite hydrogels was measured at $20 \pm 2{ }^{\circ} \mathrm{C}$, using a gravimetric method..$^{24,25}$ The dried sample of composite hydrogel was placed in distilled water $(25 \mathrm{~mL})$ and weighed after removing the surface water with filter paper at different intervals, from 0 to 30 hours. The $S_{\mathrm{g} / \mathrm{g}}$ was calculated as:

$$
S_{\mathrm{g} / \mathrm{g}}=\frac{W-W_{0}}{W_{0}}
$$

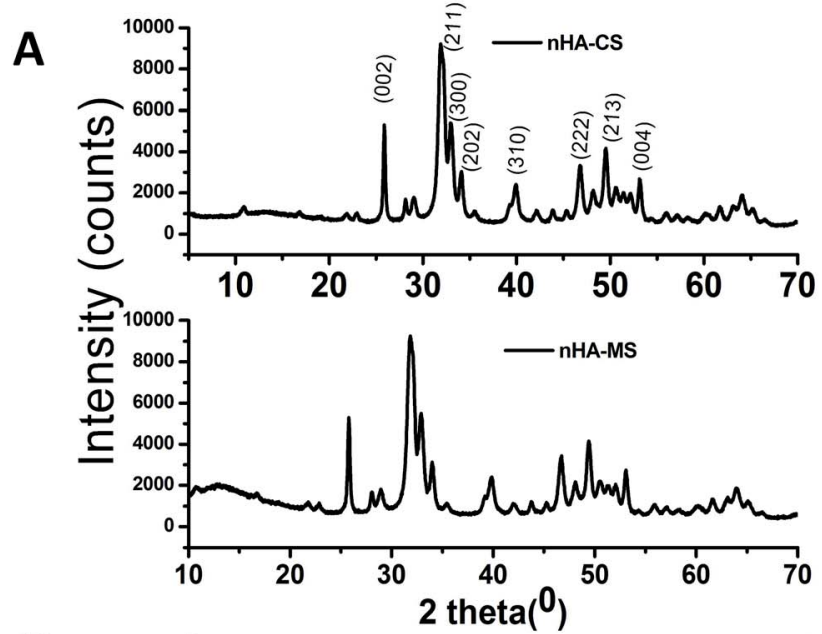

B

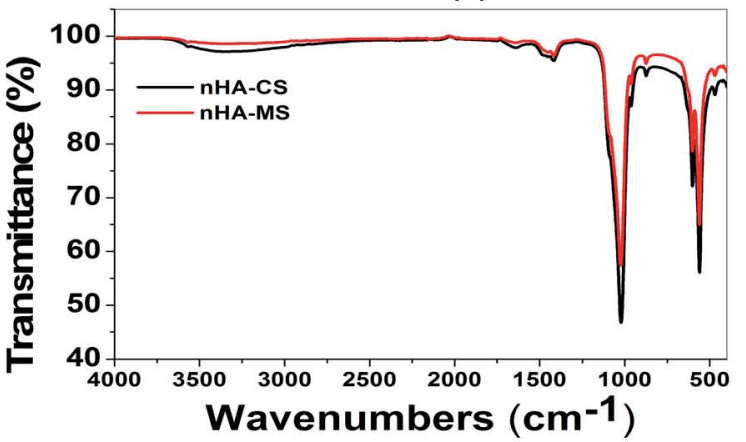

C

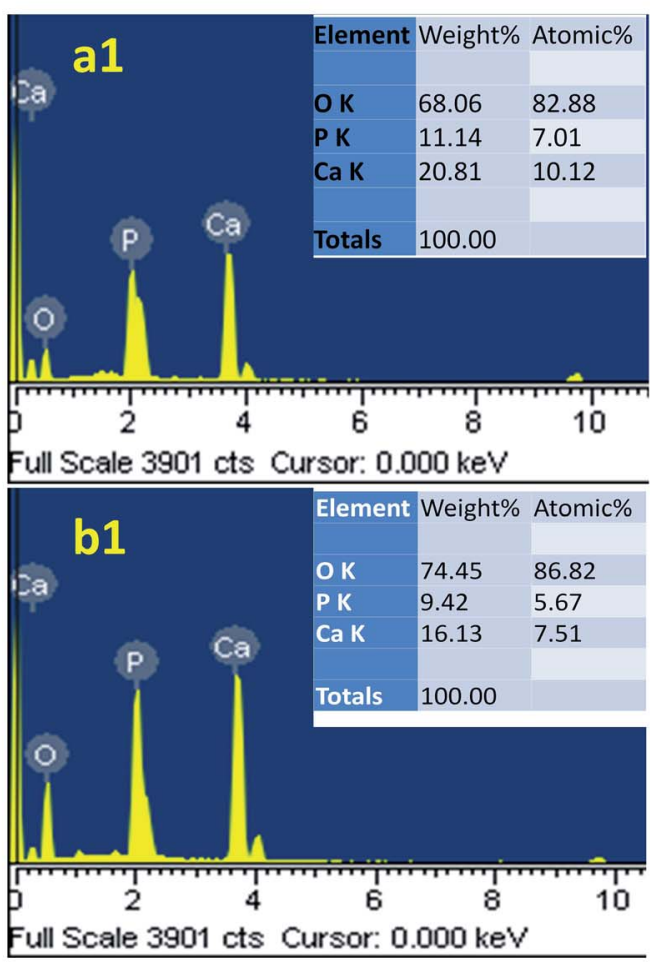

Fig. 1 (A) XRD, (B) FTIR of nHAs, (C) EDS images of (a1) nHA-MS, (b1) nHA-CS respectively. 
where $W$ and $W_{0}$ are the weights of the swollen hydrogel and the initial dry hydrogel, respectively.

\section{Adsorption of Acid Blue 113}

Acid Blue 113 is an anionic water-soluble acid dye, and it was selected as the model compound to investigate the removal abilities of the nHA hydrogels. The dye stock solutions were prepared by directly dissolving the model compound $(\mathrm{AB}=$ $100 \mathrm{mg} \mathrm{L}^{-1}, \mathrm{pH}$ 6) in distilled water. $50 \mathrm{mg}$ of composite hydrogel was added to a $10 \mathrm{~mL}$ of the aqueous solutions of the dye, and was immersed for 24 hours at $20 \pm 2{ }^{\circ} \mathrm{C}$ for maximum removal of the dye. After the 24 hours, the hydrogel was removed from the swelling medium. For the $\mathrm{AB}$ dye solution, the concentration was determined by absorption at $\lambda_{\max } 566 \mathrm{~nm}$ in the UV-vis spectra, by using Shimadzu spectrophotometer UV-2600 (Japan). ${ }^{26}$ UV spectra and images were recorded at different points.

The removal efficiency of pollutant (Acid Blue 113) and specific adsorption capacity was calculated according to the following equations:

$$
\begin{gathered}
\text { Removal efficiency }(\%)=\left(C_{0}-C_{\mathrm{e}}\right) / C_{0} \times 100 \\
\text { Adsorption capacity }\left(\mathrm{mg} \mathrm{g}^{-1}\right)=\left(C_{0}-C_{\mathrm{e}}\right) / W \times V
\end{gathered}
$$

where $C_{0}\left(\mathrm{mg} \mathrm{L}^{-1}\right)$ and $C_{\mathrm{e}}\left(\mathrm{mg} \mathrm{L}^{-1}\right)$ are the initial and equilibrium concentrations at the time of measurement of the pollutants, respectively; $V$ (L) denotes the volume of the pollutants solution, and $W(\mathrm{~g})$ is the hydrogel composites mass.

\section{Kinetic adsorption process}

The AB absorption mechanisms of nHA based hydrogels were studied employing pseudo-first-order and second-order dynamic methods. Lagergren's linear equation for the pseudofirst-order is written as

$$
\ln \left(q_{\mathrm{e}}-q_{t}\right)=\ln q_{\mathrm{e}}-k_{1} t
$$

Hos linear equation of the pseudo-second-order is written as

$$
\frac{t}{q_{t}}=\frac{1}{k_{2} q_{e}{ }^{2}}+\frac{1}{q_{e}} t
$$

where $q_{\mathrm{e}}\left(\mathrm{mg} \mathrm{g}^{-1}\right)$ and $q_{t}\left(\mathrm{mg} \mathrm{g}^{-1}\right)$ are the removal capacity of pollutants at equilibrium and time $t(\mathrm{~h})$ respectively. $k_{1}(1 / \mathrm{h})$ and $k_{2}\left(\mathrm{~g} \mathrm{mg}^{-1} \mathrm{~h}^{-1}\right)$ are the velocity constant of first-order kinetics and second-order, respectively.

\section{Results and discussion}

\section{Structural studies}

In this investigation, the wet chemical precipitation method was used to generate the nHA-CS and nHA-MS. XRD is an important analytical technique to determine the crystal structure, and its data gives information about the nHA peak
A

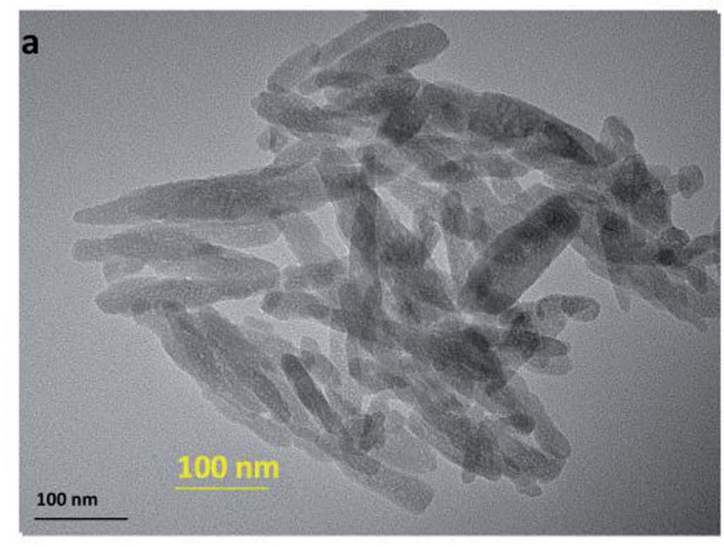

B

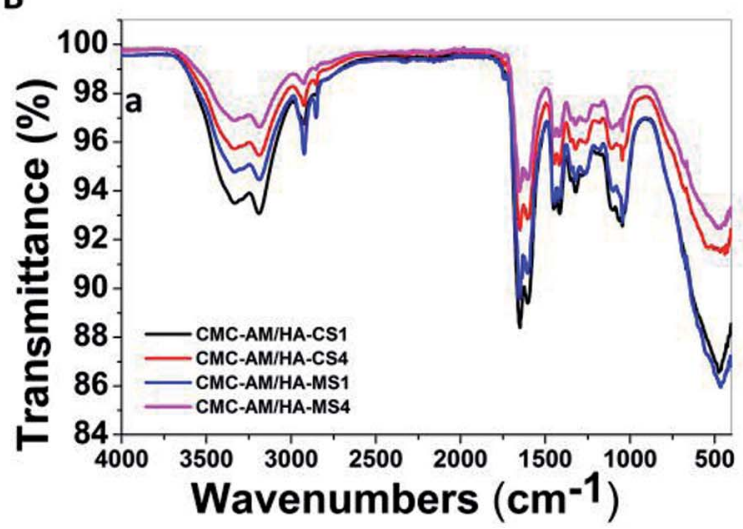

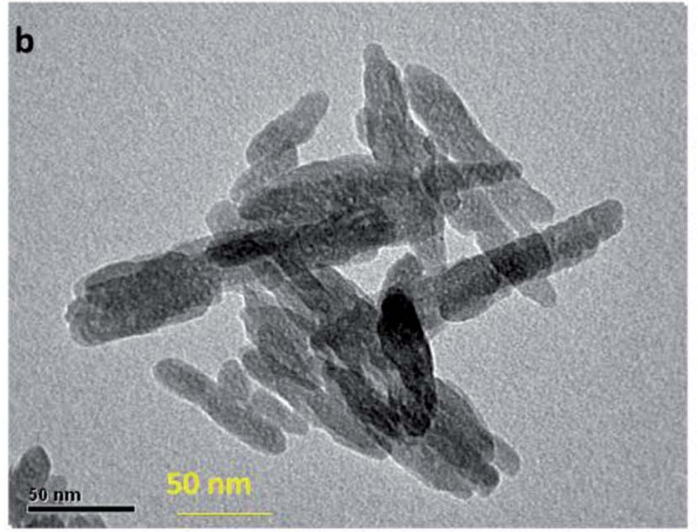

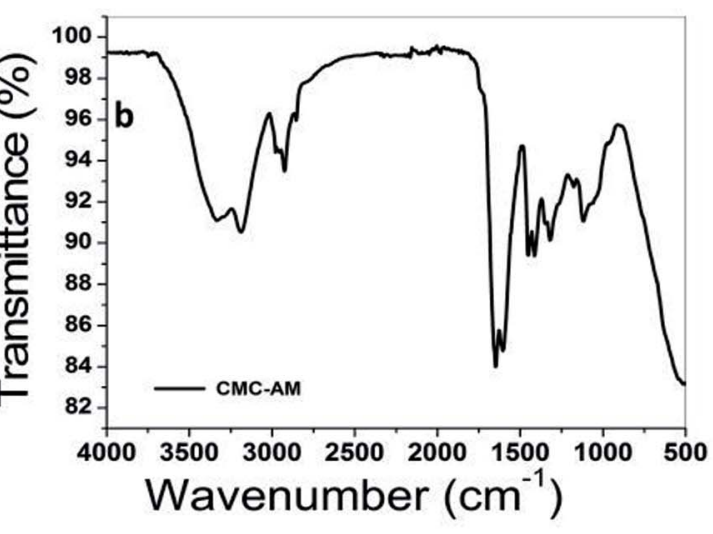

Fig. 2 (A) TEM image of $\mathrm{nHAs}$ and (B) FTIR of the $\mathrm{nHAs}$ composite hydrogels. 

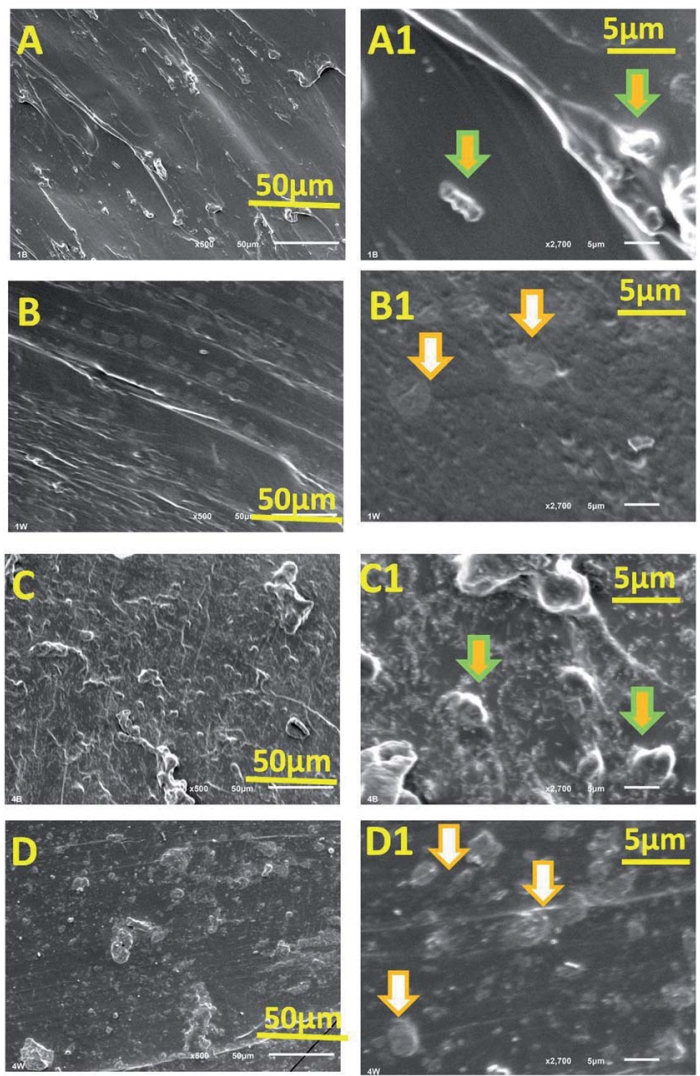

Fig. 3 SEM images of (A and A1) CMC-AM/nHA-MS1, (B and B1) CMC$\mathrm{AM} / \mathrm{nHA}-\mathrm{CS1}$, (C and C1) CMC-AM/nHA-MS4 and (D and D1) CMCAM/nHA-CS4 hydrogels.

position and intensity. The peak position and intensity depends on the nHA physical and chemical properties. Fig. 1A shows the XRD patterns of the nHA-CS and nHA-MS nanoparticles. These two nanoparticle samples exhibit similar XRD spectral peaks. The XRD patterns display strong reflections peaks at $2 \theta=25.77$, $31.87,33.01,34.14,39.98,46.80,49.55$ and $53.12^{\circ}$ corresponding to (002), (300), (202), (310), (222), (213), and (004) planes of nHA samples, respectively. The diffraction peaks can be attributed to pure nHA of CS and MS (JCPDS card no: 09-0432) confirming the formation of nHA of CS and MS. ${ }^{27}$

The ATR-FTIR spectra of nHA-CS and nHA-MS dry powder samples are shown in Fig. 1B. The transmission band at $3330.60 \mathrm{~cm}^{-1}$ and $609 \mathrm{~cm}^{-1}$ occur due to the stretching and bending vibrations of hydroxyl group present in the nHA-CS and nHA-MS. ${ }^{14}$ The supplementary bands at 1023.21, 957.90, 559.78, and $459 \mathrm{~cm}^{-1}$ were attributed to the phosphate $\left(\mathrm{PO}_{4}{ }^{3-}\right)$ anion present in the samples. ${ }^{14,28}$ Carbonate $\left(\mathrm{CO}_{3}{ }^{2-}\right)$ anion impurity bands in nHA were also noticed at 1474.17, 1415.88 and $874.63 \mathrm{~cm}^{-1}$. $^{28-30}$

ESI $1 \uparrow \uparrow$ displays the physical morphology of nHA-MS and nHA-CS which demonstrates dominant cloudlike (S1a $\uparrow$ ) and dominant cloud with a few layers (S1b $\dagger$ ) under SEM. Further, their EDS analysis indicates $\mathrm{Ca}, \mathrm{O}$, and $\mathrm{P}$ elemental presence (Fig. 1C). This data also confirmed that nHA-CS (Fig. 1C(b1)) has less $\mathrm{Ca}^{2+}$ and $\mathrm{P}$ elemental compared to nHA-MS (Fig. 1C(a1)). Similarly, ASS results indicated that nHA-MS has a higher calcium content of $40.3 \% \mathrm{p} / \mathrm{p}$, while nHA-CS has a calcium content of $38.2 \% \mathrm{p} / \mathrm{p}$. Furthermore, the size and shape of the nHA-MS and nHA-CS in TEM is exhibited as flowery flake-like structure (Fig. 2A). Similar morphology was observed in previous reports. ${ }^{28,31}$ These nanostructures have an average length and width $\sim 70 \mathrm{~nm}$ and $\sim 12 \mathrm{~nm}$ (nHA-MS, Fig. 2A(b)) and $\sim 80 \mathrm{~nm}$ and $\sim 20 \mathrm{~nm}$ (nHA-CS, Fig. $2 \mathrm{~A}(\mathrm{a})$ ).

The poly(carboxymethylcellulose-acrylamide)/nano-hydroxyapatites of a Clam shell (CMC-AM/nHA-CS) and poly(carboxymethylcellulose-acrylamide)/nano-hydroxyapatites of Magellan shells (CMC-AM/nHA-MS) hydrogels with variation in the concentration of nHA-CS and nHA-MA were prepared via a free radical polymerization process. The nHA based hydrogels were characterized by ATR-FTIR, XRD and SEM/EDS to obtain evidence of the incorporation of nHA, CMC, and AM. Here, an ATR-FTIR spectrum was used to determine the possible chemical interaction between the nHA and CMC-AM hydrogels. Fig. 2B shows the characteristics of the ATR-FTIR of the CMCAM/nHA-CS $x$, CMC-AM/nHA-MS $x(x=1,4)$ and CMC-AM hydrogels. In the CMC-AM hydrogel (Fig. $2 \mathrm{~B}(\mathrm{~b})$ ), the transmittance peaks observed at 3332.72 and $3184.43 \mathrm{~cm}^{-1}(-\mathrm{NH})$, $2927.41 \mathrm{~cm}^{-1}\left(-\mathrm{CH}_{2}\right), 1642.28$ and $1602.74 \mathrm{~cm}^{-1}(\mathrm{C}=\mathrm{O} \&-\mathrm{N}-\mathrm{H}$, amide I \& amide II groups) due to the presence of various functional groups in CMC-AM hydrogel. The additional peaks found at 1449.51, 1409.97, $1311.12 \mathrm{~cm}^{-1}\left(-\mathrm{CH}_{2}\right.$ scissoring, $-\mathrm{COO}$ symmetrical stretching, $-\mathrm{CH}_{2}$ twisting) and $1113.40 \mathrm{~cm}^{-1}$ (- $\mathrm{CH}-\mathrm{O}-\mathrm{CH}_{2}$ units) corresponds to the $\mathrm{CMC}$ hydrogel. In the case of nHA-CS and nHA-MS based hydrogels (Fig. $2 \mathrm{~B}(\mathrm{a})$ ), the peaks of nHA found at 1047.50 and $464 \mathrm{~cm}^{-1}$ due to the phosphate $\left(\mathrm{PO}_{4}{ }^{3-}\right)$ anion, which indicate the presence of nHA in hydrogels. The ATR-FTIR spectra of CMC-AM/nHA-CS and CMCAM/nHA-MS hydrogels (ESI S2A $\dagger$ ) showed differences in intensity from the neat hydrogels and nHA increase in the hydrogels. The transmittance of the hydrogels decreased (CMC-AM/nHACS1 > CMC-AM/nHA-CS4 and CMC-AM/nHA-MS1 > CMC-AM/ nHA-MS4) which might be attributed to the results of the intra-hydrogen bonds interaction between nHA and CMC-AM hydrogel. However, this phenomenon indicates that the functional groups of the nHA have possible intra-hydrogen interactions with polymeric hydrogels, which can enhance their applicability in absorption studies. A similar phenomenon observed in the XRD studies of nHA based hydrogels (ESI S2B $\dagger$ ). From the XRD results, it was noted that a high content of nHA based hydrogels exhibited in a low-intensity mountain, when compared to the low content of nHA based hydrogels, which indicates that the crystalline structure of nHA is well stabilized in CMC-AM hydrogels.

A distinct change in morphology of composite hydrogels was observed in SEM studies (Fig. 3). CMC-AM/nHA-MS1 (Fig. 3A and A1) and CMC-AM/nHA-CS1 (Fig. 3B and B1) hydrogels showed a CMC-AM network with stabilized nHA-MS and nHACS nanoparticles (indicated with white arrows). These reveal the surface morphology of hydrogel structures. A higher number of nanoparticles were grown on the surfaces of CMCAM/nHA-MS4 (Fig. 3C and C1) and CMC-AM/nHA-CS4 (Fig. 3D and D1) hydrogels. The high concentrations of nHA were stabilized with the functional groups of CMC-AM network of the 

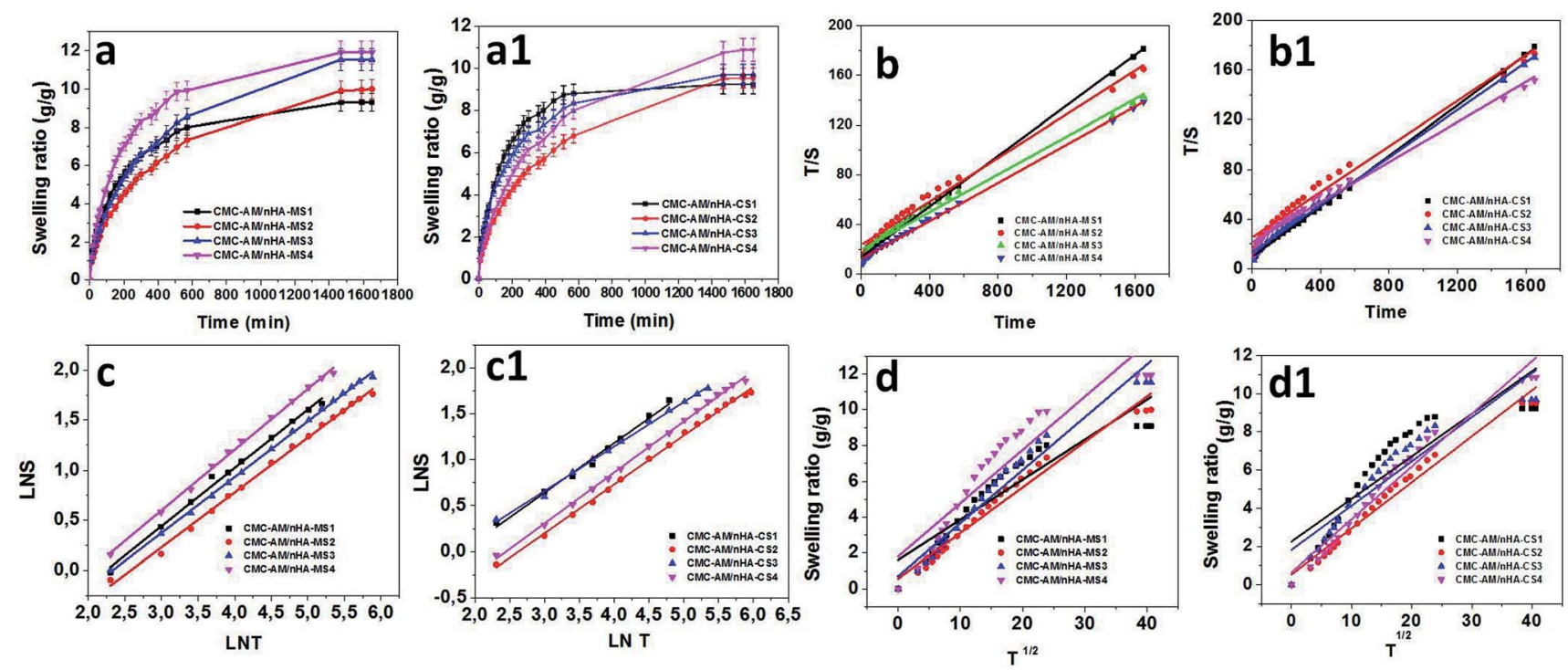

Fig. 4 Influence of nHA content on swelling behaviour of CMC-AM/nHA-CS and CMC-AM/nHA-CS hydrogels: (a and a1) swelling ratio, (b and b1) time/swelling ratio (T/S) and time ( $T$ ), (c and $c 1$ ) $\log S$ and $\log T$ and (d and d1) $S$ and $T^{1 / 2}$ graph of nHA based CMC-AM hydrogels.

hydrogel. Additionally, it was observed that the swelling mechanism of the nHA hydrogels was affected by the hydrogels structure and swelling media.

\section{Swelling behaviour of hydrogels}

Fig. 4 presents the swelling behaviour of the CMC-AM/nHA-CS $x$ and CMC-AM/nHA-MSx $(x=1-4)$ hydrogels. It was observed that there was an increase in the nHA-CS and nHA-MS concentration in the hydrogels (Fig. 4a and a1), and the water absorption capacity of the hydrogels increased. This phenomenon occurred due to the electrostatic repulsions of the hydrophilic CMC carboxylic functional groups and mainly nHA functional groups (calcium, hydroxyl, and phosphate). ${ }^{32,33}$ The functional group $\left(\mathrm{Ca}^{2+}\right)$ of the nHA can easily interact with water molecules via hydrogen and ionic interactions which leads to the higher water uptake capacity. Therefore, increases in the nHA concentration $\left(\mathrm{Ca}^{2+}\right.$ concentration in $\left.\mathrm{nHA}\right)$ in the hydrogels network are directly related to the increase of the hydrogels water absorption. Similar results reported by Du et al. ${ }^{34}$ explained that nano-hydroxyapatite concentration could increase the hydrogels swelling behaviour. He specified that more nano-hydroxyapatite creates higher osmotic pressure between the hydrogel network and swelling media. However, CMC-AM/nHA-CS hydrogels show lower swelling capacity than CMC-AM/nHA-MS hydrogels. The case is that nHA-CS has a lower percent of $\mathrm{Ca}^{2+}$ compared with nHA-MS, which is evident in the EDS analysis. Therefore, it was observed that increases of $\mathrm{Ca}^{2+}$ ions in the nHA increases the swelling capacity of the CMC-AM hydrogels.

The kinetics of water absorption process is the vital importance for determining the diffusion of water molecules into the hydrogels. The swelling and diffusion kinetic parameters of CMC-AM/nHA-CS $x$ and CMC-AM/nHA-MS $x(x=1$ to 4$)$ hydrogels were calculated from the dynamic $S_{\mathrm{g} / \mathrm{g}}$ values.
The swelling kinetics of the composite nHA hydrogels were studied according to previous reports. ${ }^{17}$ To investigate the controlling mechanism of the sorption systems experimental data were fitted with several swelling kinetic models. Here, the swelling diffusion kinetics was calculated by the following equation:

$$
\mathrm{d} S / \mathrm{d} t=k_{\mathrm{s}}\left(S_{\mathrm{eq}}-S\right)^{2}
$$

where $S_{\text {eq }}, S$, and $k_{\mathrm{s}}$ are the equilibrium swelling, the swelling at any time $t$ and the kinetic rate constant of selling, respectively. The combination of the equation two over the limits: $S=0$ at time $t=0$ and $S=S_{\text {eq }}$ at equilibrium time $t=t$, gives the following equation.

$$
\frac{t}{s}=A+B_{t}
$$

where $t$ is time, $S$ is swelling at $t, A=1 / k_{\mathrm{S}} S_{\text {eq }}^{2}=1 /(\mathrm{d} S / \mathrm{d} t)_{0}$ is the initial swelling rate of the nHA based hydrogel composites and $B=1 / S_{\text {eq }}$ is the inverse of the maximum swelling and $k_{\mathrm{s}}$ is the swelling rate constant. In order to observe the above kinetic model for these hydrogels, the graphs of $t / S$ versus $t$ (time) plotted and the initial rate of swelling $\left(r_{\mathrm{i}}\right)$, swelling rate the constant $\left(k_{\mathrm{s}}\right)$ and the theoretical equilibrium swelling $\left(T S_{\text {eq }}\right)$ values of the hydrogel composites were calculated from the slopes and the intersections of the lines obtained from the graphs (Fig. 4b and b1). The results are shown in Table 1. Table 1 shows that the values of the $T S_{\text {eq }}$ of the nHA based hydrogels are more close to an original swelling value of the nHA hydrogels. It was concluded that the swelling phenomena of the nHA hydrogels was directly related to the composition (structure) of the hydrogels. ${ }^{35}$

The dynamics of the water sorption method were investigated by monitoring the change in the amount of water imbibed by the hydrogel at various intervals. In the present study, the 

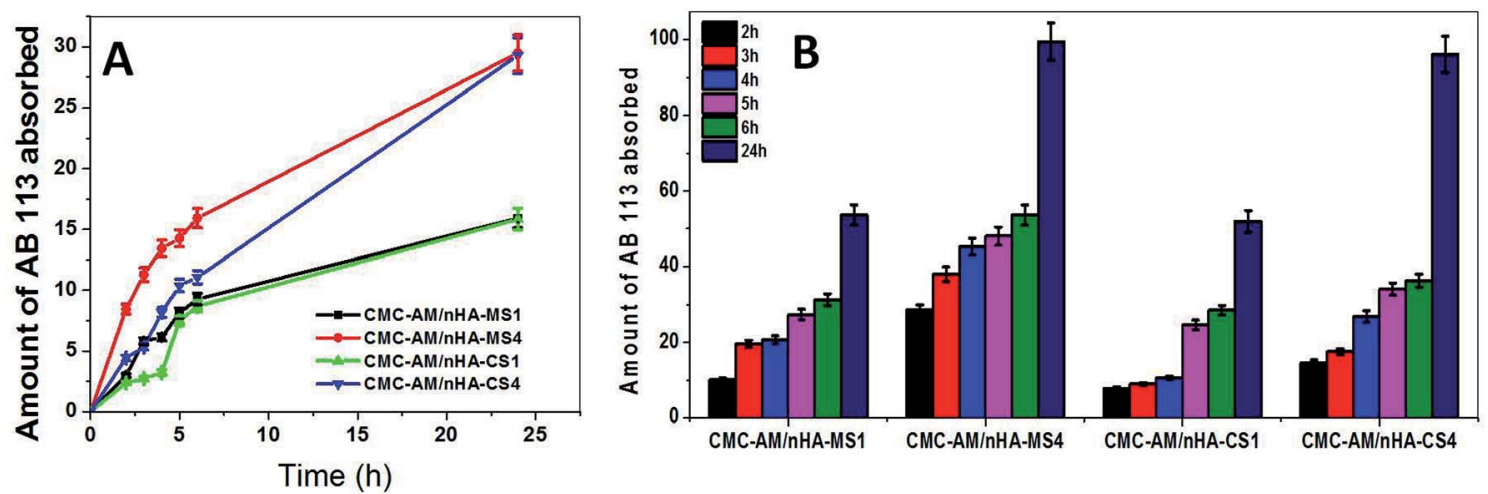

Fig. 5 (A) $A B$ adsorption capacity with time for $n H A$ varied hydrogels and (B) $A B$ removal rate by $n H A$ based hydrogels.

previous swelling results were employed. For the kinetic analysis, the swelling results obtained were utilized, and only up to $60 \%$ of the swelling curves. The swelling mechanism of the nHA hydrogels were calculated from the following equation.

$$
\text { Swelling ratio }(s)=\left(W_{\mathrm{s}}-W_{\mathrm{d}}\right) / W_{\mathrm{d}}=k t^{n}
$$

where $W_{\mathrm{s}}$ is the weight of the swollen hydrogels at time $t$, and $W_{\mathrm{d}}$ is the weight of the dry hydrogel at time 0 (first hydrogel). The constant $k$ is the characteristic of the hydrogel system, and $n$ is the swelling exponent, which indicates the water transport mechanism. When $n=0.45-0.5$ (cylindrical shape hydrogels), the release is perfect Fickian in nature, and it is the diffusion-controlled mechanism, whereas values of $n$ between 0.5 to 1.0 indicate non-Fickian (anomalous) diffusion. In anomalous diffusion, the diffusion and relaxation are said to be isochronally effective. If the $n$ value is exactly equal to unity (1), then the diffusion is designated as Case II diffusion. In very few cases, the " $n$ " value is found to exceed unity and is called super Case II diffusion $(n>1)$. To estimate the " $n$ " by using the above equation up to $60 \%$ of the swelling values, "In $S$ " versus "In $t$ " graphs were plotted to obtain the straight line. The swelling exponents were calculated from the slope of the line of $\ln S-\ln t$ plots (Fig. 4c and c1). The CMC-AM/nHA-MS and CMC-AM/nHA-CS hydrogels have $n$ value between 0.5468 to 0.60594 and 0.49464 to 0.5531 , respectively. These values (over 0.5 ) specify that the swelling transport mechanism of the nHA based hydrogels were non-
Fickian diffusion or anomalous transport, which indicates that relative rates of diffusion and CMC-AM relaxation are comparable. $^{36}$

The diffusion coefficients $(D)$ of the composite hydrogels were calculated via using the short time approximation method. This method is valid for the first $60 \%$ of the swelling results. The hydrogels diffusion coefficients were calculated by using the following equation. ${ }^{35}$

$$
S=4\left[D / \pi r^{2}\right]^{1 / 2} t^{1 / 2}
$$

where $r, S$, and $t$ represent the radius of the nHA hydrogel, swelling ratio and time, respectively. To investigate the $D\left(\mathrm{~cm}^{2}\right.$ $\mathrm{s}^{-1}$ ) of hydrogels, $S$ versus $t^{1 / 2}$ were plotted and the $D$ values were calculated from the slopes of these lines (Fig. $4 \mathrm{~d}$ and $\mathrm{d} 1$ ). The kinetic parameters of the data obtained are presented in Table 1. The diffusion coefficient increased with increasing nHA content in the hydrogels which affect the diffusivity of the hydrogels. The values of the $D$ of the CMC-AM/nHA-CS and CMC-AM/nHA-MS hydrogels varied from $0.22353-0.27681$ and $0.22377-0.29737 \mathrm{~cm}^{2} \mathrm{~s}^{-1}$, respectively. From the results, it was observed that CMC-AM/nHA-MS hydrogels have higher $D$ values than nHA-CS based hydrogels. Therefore, nHA-MS based hydrogel composites have a higher water absorption capacity.

\section{Dye removal}

The removal of dye from polluted water is an important issue global. In this investigation, CMC-AM/nHA-CS $x$ and CMC-AM/

Table 2 Adsorption kinetic parameters of the pseudo first order and pseudo second-order models for the removal of AB dye by $\mathrm{CMC}$-AM/nHA hydrogels at $25^{\circ} \mathrm{C}$

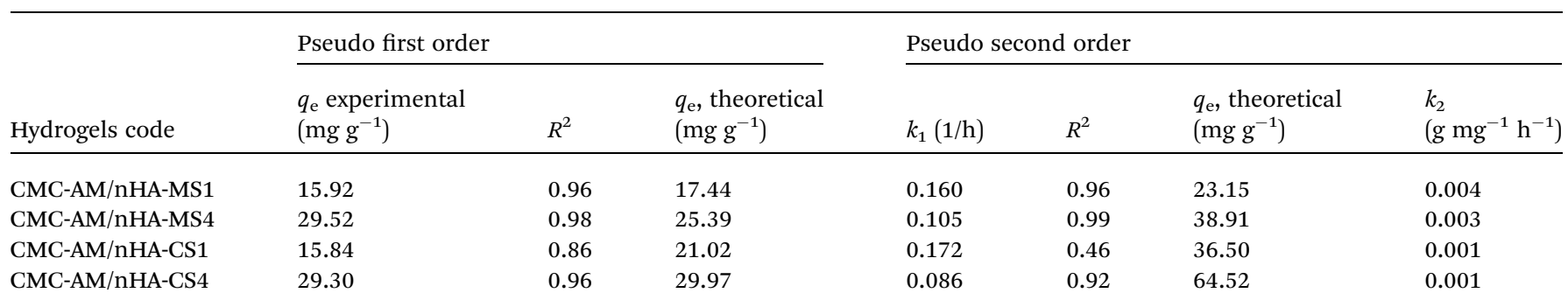


nHA-MS $x(x=1,4)$ hydrogels were selected to study the effect of the nHA-CS and nHA-MS concentration of the hydrogels on the $\mathrm{AB}$ adsorption capacities. The adsorptions of $\mathrm{AB}$ on selected hydrogels are shown in Fig. 5. These results explained that the adsorption of $\mathrm{AB}$ increased with the increase of nHA content in the CMC-AM hydrogels and a higher removal rate was found in the case of CMC-AM/nHA-MS $x$ hydrogels when compared to CMC-AM/nHA-CSx. A similar phenomenon was observed in swelling studies. As shown in Fig. $5 \mathrm{~B}$, the (\%) removal rate of $\mathrm{AB}$ is 51.95, 53.63, 96.08 and 99.44\% with CMC-AM/nHA-CS1, CMCAM/nHA-MS1, CMC-AM/nHA-CS4 and CMC-AM/nHA-MS4 composite hydrogels as adsorbents, respectively.

The AB adsorption capacities of the CMC-AM/nHA-CS $x$ and CMC-AM/nHA-MS $x(x=1,4)$ hydrogels are presented in Fig. 5. As shown in Fig. 5A, the CMC-AM/nHA-CS1, CMC-AM/nHA-CS4, CMC-AM/nHA-MS1 and CMC-AM/nHA-MS4 hydrogels have AB adsorption capacities of 15.84, 29.30, 15.92 and $29.52 \mathrm{mg} \mathrm{g}^{-1}$, respectively. However, the adsorption capacity of CMC-AM/ nHA-MS4 was much higher than that of other hydrogels. This behaviour mainly attributed to two reasons: (i) the percent of $\mathrm{Ca}^{2+}$ and $\mathrm{P}$ elements in nHA and concentration of nHA in the hydrogels. It was confirmed from structural analysis, and it is shown in Fig. 1D, 2B and 3 and (ii) the swelling ratio (Fig. 4D) of hydrogels. Recently, several researchers have reported various kinds of sorbent materials for removing the $\mathrm{AB}$ dye from water. For example, sodium hydroxide treated fallen leaves of Prunus dulcis showed $25.51 \mathrm{mg} \mathrm{g}^{-1}$ of $\mathrm{AB}$ dye adsorption and CTAB modified leaves of Prunus dulcis showed $97.09 \mathrm{mg} \mathrm{g}^{-1}$ adsorption capacity. ${ }^{2}$ Gupta et al. reported that the waste of rubber tire based activated carbon sorbent showed $9.72 \mathrm{mg} \mathrm{g}^{-1}$ adsorption capacity of $\mathrm{AB}$ dye. ${ }^{37}$ Similarly, alkali-treated sawdust sorbent materials adsorbed $24.39 \mathrm{mg} \mathrm{g}^{-1}$ of dye. ${ }^{38}$ The adsorption capacity of the nHA based CMC-AM hydrogels has better results when compared to a few reports. The increase in the adsorption capability is probably due to the higher interaction between nHA and CMC-AM hydrogels and mainly due to nHA content. However, the use of nHA based CMC-AM hydrogel adsorbents for dye removal is very promising since natural polymer and nHA are low cost and available in many places worldwide.

\section{Adsorption kinetics}

A kinetic study of $\mathrm{AB}$ adsorption on the developed nHA hydrogels was performed. The $\mathrm{AB}$ sorption capacity of all samples increased rapidly (Fig. 5A), around $50 \%$ of the total sorbed dye occurred within the first 6 hours. The results obtained from the pseudo-first-order and pseudo-second-order models kinetics models (Table 2) reveal that the absorption process of $\mathrm{AB}$ into CMC-AM/nHA-CS and CMC-AM/nHA-MS composite hydrogels were better described via the pseudo-first-order kinetic model since the experimental data was best correlated with eqn (9) and its $R^{2}$ is closer to 1 . Additionally, the " $q_{\mathrm{e}}$ " values obtained from the pseudo-first-order kinetic model were more consistent with experimental " $q_{\mathrm{e}}$ " values when compared to pseudo-secondorder model values (Table 2). This kind of phenomena was also observed in a previous report. ${ }^{39}$ The pseudo-first order is based on the assumption that the rate-limiting step may be chemical sorption. ${ }^{40}$ However, in the case of pseudo-first-order and pseudo-second-order models, $R^{2}$ values increased with nHA-CS and nHA-MS content in the CMC-AM hydrogels.

\section{Conclusion}

This study has produced two different nHA based CMC-AM hydrogels, synthesized by the free-radical polymerization process. The nHA synthesized from waste Clam shells and Magellan shells by the wet chemical precipitation method, respectively. ATR-FTIR, XRD and SEM studies revealed the formation of the nHA. From the EDS and AAS analysis, the percentage of $\mathrm{Ca}^{2+}$ was higher in nHA-MS which improved the swelling behaviour of the nHA hydrogels. However, both composite hydrogels developed followed non-Fickian diffusion transport mechanism. Further, it was improved by the AB dye absorption capacity. $\mathrm{AB}$ absorption studies were conducted using the nHA based hydrogels, which have suggested that the higher nHA contained (CMC-AM/nHA-MS4 and CMC-AM/nHACS4) can be used as a possible candidate for the removal of dye. The experimental data fit well with the pseudo-first-order model kinetic model; based on the $R^{2}$ (closed to 1 ) values and experimental " $q$ e" values. These nHA based CMC-AM hydrogels have the potential to be employed as a suitable adsorbent for the removal of pollutants in contaminated water. Moreover, the proposed nHA based composite hydrogel sorbents were developed from waste shells, which have low cost and a widely available material for the removal of dyes from contaminated water.

\section{Conflicts of interest}

The authors declare no competing financial interest.

\section{Acknowledgements}

The authors, Kokkarachedu Varaprasad and Dariela Nunez, wish to acknowledge the Grant R16F10013 (CONICYT regional), PAI 7815020005 (DN), Fondecyt 11160073 (KVP) and Centro de Investigación de Polímeros Avanzados (CIPA), CONICYT Regional, GORE BIO-BIO, R17A10003. Dr Yallapu acknowledges K22 CA174841 and 1R15CA213232 Award from NIH.

\section{References}

1 V. K. Gupta, J. Environ. Manage., 2009, 90, 2313-2342.

2 S. N. Jain and P. R. Gogate, J. Environ. Chem. Eng., 2017, 5, 3384-3394.

3 K. Varaprasad, T. Jayaramudu and E. Rotimi, Carbohydr. Polym., 2017, 164, 186-194.

4 J. Zhou, B. Hao, L. Wang, J. Ma and W. Cheng, Sep. Purif. Technol., 2017, 176, 193-199.

5 R. Sahraei, Z. S. Pour and M. Ghaemy, J. Cleaner Prod., 2017, 142, 2973-2984.

6 S. Sood, V. Kumar, S. Agarwal, K. Dev and D. Pathania, Int. J. Biol. Macromol., 2017, 101, 612-620. 
7 M. R. Rezaei, M. Hashemi, C. L. Coz and V. Coma, Food Hydrocolloids, 2017, 70, 36-45.

8 A. Esmaeili and M. Haseli, Mater. Sci. Eng., C, 2017, 77, 11171127.

9 K. Varaprasad, G. Malegowd, T. Jayaramudu, M. Mohan and R. Sadiku, Mater. Sci. Eng., C, 2017, 79, 958-971.

10 Y. Shen, X. Bo, Z. Tian, Y. Wang, X. Guo, M. Xie, F. Gao, M. Lin, X. Guo and W. Ding, Green Chem., 2017, 19, 26462652.

11 X. Guo, L. Yu, L. Chen, H. Zhang, L. Peng, X. Guo and W. Ding, J. Mater. Chem. B, 2014, 2, 1760-1763.

12 Y. Azis, N. Jamarun, S. Arief and H. Nur, Orient. J. Chem., 2015, 31, 1099-1105.

13 C. A. Papadimitriou, G. Krey and N. Stamatis, J. Chem. Technol. Biotechnol., 2017, 92, 1943-1947.

14 Y. Xie, W. He, F. Li, T. Shalika, H. Perera, L. Gan, Y. Han, X. Wang, S. Li and H. Dai, ACS Appl. Mater. Interfaces, 2016, 8, 10212-10219.

15 C. Chang, N. Peng, M. He, Y. Teramoto, Y. Nishio and L. Zhang, Carbohydr. Polym., 2013, 91, 7-13.

16 A. Szcze, L. Ho and E. Chibowski, Adv. Colloid Interface Sci., 2017, 249, 321-330.

17 A. Shavandi, A. E. A. Bekhit, A. Ali and Z. Sun, Mater. Chem. Phys., 2015, 149-150, 607-616.

18 S. Kocaman, G. Ahmetli, A. Cerit, A. Yucel and M. Gozukucuk, International Journal of materials and metallurgical engineering, 2016, 10, 438-441.

19 J. F. Cawthray, A. L. Creagh, C. A. Haynes and C. Orvig, Inorg. Chem., 2015, 54, 1440-1445.

20 H. El Khal and N. H. Batis, New J. Chem., 2015, 39, 35973607.

21 S. H. Jang, Y. G. Jeong, B. G. Min, W. S. Lyoo and S. C. Lee, J. Hazard. Mater., 2008, 159, 294-299.

22 H. Bundela and A. K. Bajpai, eXPRESS Polym. Lett., 2008, 2, 201-213.

23 H. Hou, R. Zhou, P. Wu and L. Wu, Chem. Eng. J., 2012, 211212, 336-342.
24 T. Jayaramudu, G. M. Raghavendra, K. Varaprasad, R. Sadiku, K. Ramam and K. M. Raju, Carbohydr. Polym., 2013, 95, 188-194.

25 T. Jayaramudu, G. M. Raghavendra, K. Varaprasad, R. Sadiku and K. M. Raju, Carbohydr. Polym., 2013, 92, 2193-2200.

26 W. A. Khanday, M. Asif and B. H. Hameed, Int. J. Biol. Macromol., 2016, 95, 895-902.

27 H. C. Shum, A. Bandyopadhyay, S. Bose and D. A. Weitz, Chem. Mater., 2009, 81, 5548-5555.

28 Y. Wang, X. Ren, W. Su, Y. Zhang, X. Sun and X. Li, Cryst. Growth Des., 2015, 15, 1949-1956.

29 K. Ronan and M. B. Kannan, ACS Sustainable Chem. Eng., 2017, 5, 2237-2245.

30 S. David Cifrulak, Am. Mineral., 1970, 55, 185-824.

31 K. Varaprasad, M. Pariguana, G. M. Raghavendra, T. Jayaramudu and E. R. Sadiku, Mater. Sci. Eng., C, 2017, 70, 85-93.

32 A. Sand, M. Yadav and K. Behari, Carbohydr. Polym., 2010, 81, 97-103.

33 S. Saber-samandari, H. Yekta and S. Saber-samandari, Journal of Mineral, Metal and Material Engineering, 2015, 1, 19-25.

34 M. Du, W. Song, Y. Cui, Y. Yang and J. Li, J. Mater. Chem., 2011, 21, 2228-2236.

35 Y. M. Mohan, K. Sudhakar and P. S. Keshava, Int. J. Polym. Mater. Polym. Biomater., 2006, 55, 37-41.

36 A. R. Khare and N. A. Peppas, Biomaterials, 1995, 16, 559567.

37 V. K. Gupta, B. Gupta, A. Rastogi, S. Agarwal and A. Nayak, J. Hazard. Mater., 2011, 186, 891-901.

38 M. Ahmad, K. Megat, W. Saime, W. Ngah, S. H. Zolkafly, L. C. Teong, Z. Azran and A. Majid, J. Environ. Sci., 2012, 24, 261-268.

39 A. M. Aljeboree, A. N. Alshirifi and A. F. Alkaim, Arabian J. Chem., 2017, 10, S3381-S3393.

40 Y. S. Ho and G. Mckay, Resour., Conserv. Recycl., 1999, 25, 171-193. 